\title{
Development and optimization of ifosfamide nanostructured lipid carriers for oral delivery using response surface methodology
}

\author{
Ramaiyan Velmurugan $^{1} \cdot$ Subramanian Selvamuthukumar $^{1}$
}

Received: 18 July 2014/ Accepted: 10 March 2015/Published online: 26 March 2015

(c) The Author(s) 2015. This article is published with open access at Springerlink.com

\begin{abstract}
The research focuses on the development and optimization of ifosfamide nanostructured lipid carriers for oral delivery with the application of response surface methodology. The objectives of the study were to develop a formulation for ifosfamide to be delivered orally, overcome the instability of the drug in acidic environment during oral administration, to sustain the release, drug leakage during storage and low loading capacity. A modified solvent diffusion method in aqueous system was applied to prepare nanostructured lipid nanoparticles. Hydrophilic polymers such as chitosan and sodium alginate were used as coating materials. Glycerol mono oleate and oleic acid were used as solid and liquid lipid, respectively. Poloxamer is used as stabilizers. The central composite rotatable design consisting of three-factored factorial design with three levels was used in this study. The physiochemical characterization included evaluation of surface morphology, particle size and surface charge of the drug in the delivery system. The in vitro drug release, entrapment and drug loading efficiency and as well as the storage stability were evaluated. The results showed that the optimal formulation was composed of drug/lipid ratio of 1:3, organic/aqueous phase ratio of $1: 10$ and concentration of surfactant of $1 \% \mathrm{w} / \mathrm{v}$. Ifosfamide nanostructured lipid carrier under the optimized conditions gave rise to the entrapment efficiency of $77 \%$, drug loading of $6.14 \%$, mean diameter of $223 \mathrm{~nm}$ and zeta potential value of
\end{abstract}

Subramanian Selvamuthukumar

smk1976@gmail.com

Ramaiyan Velmurugan

ramaiyan.dr@gmail.com

1 Department of Pharmacy, Annamalai University, Annamalai Nagar 608002, Tamilnadu, India
$-25 \mathrm{mV}$. Transmission electron microscopy analysis showed spherical particles. The in vitro experiment proved that ifosfamide from the delivery system released gradually over the period of $72 \mathrm{~h}$. Sodium alginate cross-linked chitosan nanostructured lipid carrier demonstrated enhanced stability of ifosfamide, high entrapment efficiency and sustained release.

Keywords Ifosfamide - Nanostructured lipid carriers . Oral delivery $\cdot$ Response surface methodology $\cdot$ Solvent diffusion technique $\cdot$ Sustained release

\section{Introduction}

Ifosfamide is one of the widely used antineoplastic drugs belonging to the alkylating agents group. Ifosfamide is chemically 3-(2-chloroethyl)-2-[(2-chloroethyl) amino]-tetrahydro-2H-1,3,2-oxazophosphorin-2-oxide. Ifosfamide is a white crystalline hygroscopic powder having a melting point of $40{ }^{\circ} \mathrm{C}$. The powder has a water solubility of about $100 \mathrm{mg} / \mathrm{ml}$. Ifosfamide is used in the treatment of a variety of solid tumors including those of the cervix, endometrium, lung, ovary, testes and thymus as well as in sarcoma and in the treatment of Burkitt's lymphoma. Ifosfamide, being susceptible to hydrolytic degradation degrades in acidic media with its rate of degradation depending on the $\mathrm{pH}$ of the solution; it is commercially available in dry form and is supplied as sterile packaged dry powder for dissolution in water for injection prior to administration. However, the low melting point and the hygroscopic nature of ifosfamide make it necessary to fill the powder with great care by accurately controlling both temperature and humidity to achieve a sterile product. Further, prolonged storage of the dry powder also results in sintering and yellowing, which 
in turn leads to a reduction in dissolution rate, thereby increasing the time required for reconstitution (Alexander et al. 1993).

There is a need for an oral dosage form which eliminates the issues associated with the drug. Oral delivery of Ifosfamide could provide better means and its success is expected to revolutionize cancer chemotherapy. Oral delivery can maintain an optimum concentration of drug in circulation which can provide prolonged exposure to cancerous cells, which will in turn improve the efficacy and decrease the adverse effects. Oral administration of Ifosfamide would be pleasant for the patient and would no longer constitute a risk for the medical personnel. Hence, it was sought to develop an oral delivery system that could stabilize Ifosfamide in acidic environment and reduce the formation of the toxic metabolites by preventing its exposure to the acidic medium. NLCs of ifosfamide could sustain the release of, as well as overcome the instability of the drug in acidic environment during oral administration.

Lipids and lipid nanoparticles are extensively employed as oral-delivery systems for drugs and other active ingredients. Lipids usually enhance drug absorption in the gastrointestinal tract (GIT), and when formulated as nanoparticles, these molecules improve mucosal adhesion due to small particle size and increasing GIT residence time. In addition, lipid nanoparticles may also protect the loaded drugs from chemical and enzymatic degradation and gradually release drug molecules from the lipid matrix into blood, resulting in improved therapeutic profiles compared to free drug. Therefore, due to their physiological and biodegradable properties, lipid molecules may decrease adverse side effects and chronic toxicity of the drug-delivery systems when compared to other of polymeric nature.

Preparation of solid lipid nanoparticles (SLNs) is one of the approaches used to encapsulate hydrophilic drugs to obtain good sustained release and better entrapment efficiency. But, the common disadvantages experienced with solid lipid nanoparticles are particles growing, unpredictable gelation tendency, unexpected dynamics of polymorphic transitions and inherent low incorporation rate due to the crystalline structure of the solid lipid (Das and Chaudhury 2011; Muller et al. 2002a, b; Selvamuthukumar and Velmurugan 2012). In general, drug molecules stay in between the fatty acid chains or as amorphous clusters in crystal imperfections within SLN matrix. But, when lipid transforms to low energetic form, it forms a perfect crystalline lattice that allows very small space for the drug molecules. Therefore, expulsion of encapsulated drug molecules may be observed during storage, especially when SLN matrix is composed of a highly purified lipid, which leads to limited drug loading capacity of SLNs.
Therefore, an amount of entrapped drug and drug release profile of SLNs may change with storage time (Westesen et al. 1997).

In the process of further improvement and reduction of these drawbacks of SLN, nanostructured lipid carrier (NLC) has been evolved as alternative drug carrier systems. NLC matrix is composed of mixture of spatially different lipid molecules, normally mixture of solid and liquid lipid, which leads to more imperfections in the matrix to accommodate more drug molecules than SLN. Despite the presence of liquid lipid, NLC matrix is solid at room/body temperature (Chen et al. 2010). It is expected that the drug loading capacity will be enhanced, drug expulsion during storage will be minimized due to the imperfect crystal lattice and drug release profile can be easily modulated by varying the lipid matrix composition (Radtke et al. 2005a, b).

Glycerol mono oleate is a lipid that can incorporate large amounts of water in it and form liquid crystalline phases. Due to this property, it has been used for sustained release of Ifosfamide. A liquid lipid oleic acid has been used, which acts act as a stabilizer for water in oil emulsions. Poloxamer 188 was used as surfactant and sodium alginate has been used as a crosslinking agent. PVA is a stabilizer for $(\mathrm{o} / \mathrm{w})$ emulsions and was used in sonication to stabilize the emulsion. Low-molecularweight chitosan having $85 \%$ degree of deacetylation was used. Low-molecular-weight and higher degree of deacetylation are responsible for improving the solubility of chitosan. Low-molecular-weight chitosan also helps achieve smaller particle size and better redispersibiliy of particles in water. Introduction of chitosan also produces nanoparticles with positive surface charge and addition of sodium alginate changes the surface charge to a negative value. As Ifosfamide is a highly hydrophilic drug, it is very difficult to prepare a nanoformulation with high drug loading efficiency by using lipid phase alone. Therefore, chitosan coating over nanostructured lipid carriers will produce a well-dispersed nanoparticle with enhanced drug loading efficiency and modified drug-release profile.

Further, the effects of drug/lipid ratio $\left(X_{1}\right)$, organic/ aqueous phase ratio $\left(X_{2}\right)$ and surfactant concentration $\left(X_{3}\right)$ on the entrapment efficiency (EE), drug loading (DL) percentage and mean particle size (PS) of IF NLC were investigated in detail making use of central composite rotatable design (CCRD) which is an ideal tool for process optimization (Box and Hunter 1957; Kassama et al. 2008). Model equations were derived by computer simulation programming Design Expert ${ }^{\circledR}$ 8.0.7.1 to optimize Ifosfamide NLC. For a better understanding of the three variables, the models were presented as three-dimensional (3D) response surface graphs. 


\section{Materials and methods}

\section{Materials}

Ifosfamide (99.0 \% purity) was obtained from Sigma Aldrich (India). Glycerol monooleate and oleic acid were purchased from metro labs Ltd., Chennai. Lutrol ${ }^{\circledR}$ F 68 (Poloxamer 188) was kindly gifted by BASF India Ltd. Low-molecular-weight chitosan (MW 10000-12000 DA) was acquired from Aldrich Chemical Co (Mumbai, India). Ethanol, acetone and other chemicals were of analytical grade. Milli Q water (Millipore) was used throughout the studies.

\section{Preparation of NLC}

Weighed quantities of glycerol monooleate, oleic acid and Ifosfamide were dissolved in $5 \mathrm{~mL}$ of mixture of ethanol and acetone $(1: 1 \mathrm{v} / \mathrm{v})$ in a water bath at $35^{\circ} \mathrm{C}$. The resultant organic solution was quickly dispersed into $50 \mathrm{~mL}$ of aqueous solution of Poloxamer $188(1 \% \mathrm{w} / \mathrm{v})$ at room temperature $\left(25^{\circ} \mathrm{C}\right)$ under sonication for $2 \mathrm{~min}$. To this primary emulsion $6.25 \mathrm{~mL}$ of chitosan solution $(2 \% \mathrm{w} / \mathrm{v})$ (Chitosan solution was prepared by dissolving $2.4 \mathrm{~g}$ in $100 \mathrm{~mL}$ water containing $2 \% \mathrm{w} / \mathrm{v}$ acetic acid) was added and sonicated for $2 \mathrm{~min}$. Then $10 \mathrm{~mL}$ of sodium alginate solution was added under magnetic stirring for 30 min until nano suspension was obtained. Prepared NLC was placed into a vacuum desiccator for $24 \mathrm{~h}$ at room temperature to evaporate the residual organic solvent. The final product is placed in dry and cool place until further use (Pandit and Dash 2011; Yuan et al. 2007; Hu et al. 2008). A schematic representation of ifosfamide NLC preparation and nanoparticles containing chitosan and sodium alginate is given in Figs. 1 and 2, respectively.

\section{Experimental design}

Preliminary experiments indicated that the variables, such as a ratio between the drug and lipid concentration, a ratio of organic/aqueous phase volume and the concentration of surfactant were the main factors that affected the particle size, drug loading and entrapment efficiency of nanoparticles. Thus, a central composite rotatable design-response surface methodology (CCRD-RSM) was used to systemically investigate the influence of these three critical formulation variables on particle size, drug loading and entrapment efficiency of the prepared NLC. The details of the design are listed in the Table 1. For each factor, the experimental range was selected based on the results of preliminary experiments and the feasibility of preparing the NLC at the extreme values. The range of values for variables was selected as follows:
Drug/lipid ratio $\left(X_{1}\right)-1: 3-1: 7$

Organic/aqueous phase ratio $\left(X_{2}\right)-1: 5-1: 15$

Surfactant concentration $\left(X_{3}\right)-0.25-1 \%$.

With the use of three factors to be studied, CCRD-RSM suggests a total of 20 experiments to be carried out. All the formulations in these experiments were prepared in duplicate.

\section{HPLC assay method for Ifosfamide}

The HPLC separation of Ifosfamide was achieved using C18 column $(250 \times 4.6 \mathrm{~mm}, 5 \mu$; Phenomenex $)$. The mobile phase consisted of water: acetonitrile $(70: 30 \mathrm{v} / \mathrm{v})$. The flow rate was maintained at $1.5 \mathrm{~mL} / \mathrm{min}$ and the column was maintained at $40{ }^{\circ} \mathrm{C}$. The column effluents were monitored at $195 \mathrm{~nm}$ and quantified using area under the peak for the unknown drug and compared with area under the peak for the standard solutions prepared in the mobile phase (Pandit and Dash 2011).

\section{Characterisation}

Particle size, polydispersity index and zeta potential measurement

Particle size and polydispersity indices of NLC formulations were measured by dynamic light scattering using a 90 plus particle sizer (Master sizer, Malvern instruments) equipped with MAS OPTION particle sizing software. The measurements were made at a fixed angle of $90^{\circ}$ for all samples. The samples were suitably diluted with Milli Q water for every measurement. Zeta potential measurements were also made using an additional electrode in the same instrument. For zeta potential determination, samples of all formulations were diluted with $0.1 \mathrm{mM} \mathrm{KCl}$ and placed in the electrophoretic cell, where an electric field of about $15 \mathrm{~V} / \mathrm{cm}$ was applied. The mean hydrodynamic diameter (Dh) and polydispersity index (PI) of the particles were calculated using the cumulative analysis after averaging the three measurements.

\section{Transmission electron microscopy}

Transmission electron microscopy (TEM) was performed using a Philips CM 10 transmission electron microscope. The sample was prepared by a formvar resin grid method. Briefly, a $0.5 \%$ w/v suspension of NLC was sprayed on a formvar resin-coated TEM grid and air-dried for $10 \mathrm{~min}$ before observation. Contrast enhancement and particle measurement were performed using the NIH image software. 
Fig. 1 Schematic presentation of Ifosfamide NLC preparation

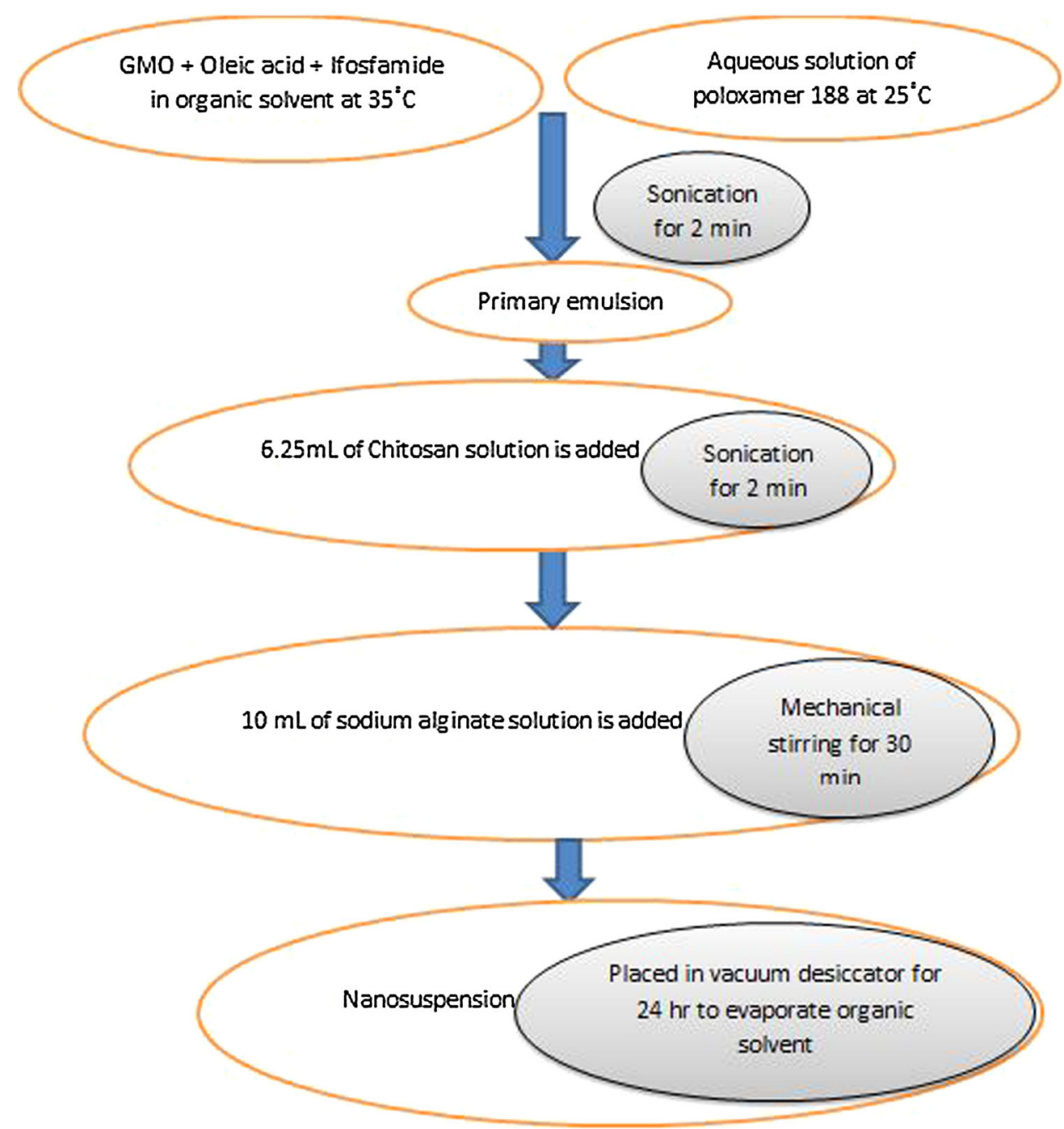

Fig. 2 Schematic diagram of nanoparticles containing chitosan and sodium alginate

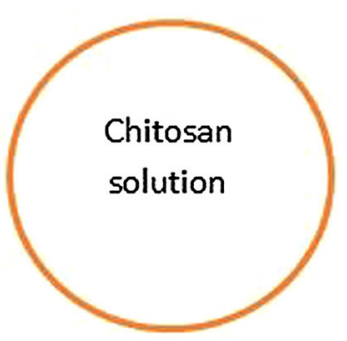

Primary emulsion

\section{Determination of drug loading and entrapment efficiency}

Drug encapsulation efficiency and drug loading of the prepared NLCs were determined by the following procedures. First, a certain volume of NLCs suspension was accurately taken, dissolved and diluted with anhydrous methanol. Then, drug content in the resultant solution was determined

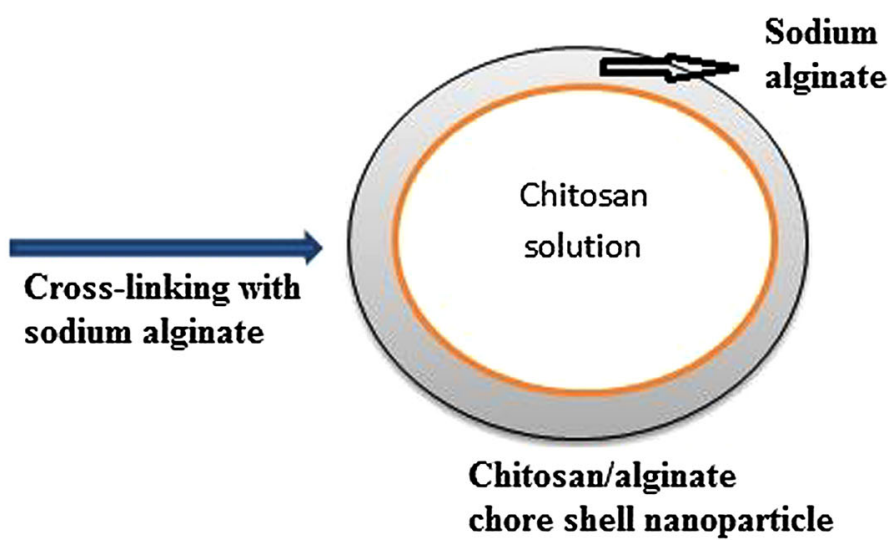

by HPLC method described in item 2.4, and the calculated drug amount was designated as $W_{\text {total }}$. To determine the unencapsulated drug, equal volume of NLC suspension was accurately taken and ultra-filtered by a filter membrane with molecular weight cut-off (MWCO) of $12 \mathrm{kDa}$ (Reili Separation Instrument Factory, Shanghai, China). The ultra-filtrate was diluted with anhydrous ethanol and drug content in 
Table 1 Independent variables and their corresponding levels of NLC preparation for CCRD

\begin{tabular}{lcllll}
\hline Variables & Levels & & & & \\
\cline { 2 - 6 } & -1.682 & -1 & 0 & +1 & +1.682 \\
\hline Drug/lipid ratio & $1: 1.64$ & $1: 3$ & $1: 5$ & $1: 7$ & $1: 8.36$ \\
$\begin{array}{l}\text { Organic/aqueous phase } \\
\text { ratio }\end{array}$ & $1: 1.59$ & $1: 5$ & $1: 10$ & $1: 15$ & $1: 18.41$ \\
$\begin{array}{l}\text { Concentration of } \\
\text { surfactant }\end{array}$ & - & $0.25 \%$ & $0.57 \%$ & $1 \%$ & $1.29 \%$ \\
\hline
\end{tabular}

the resultant solution was analyzed under the same HPLC condition. The amount of free drug was designated as $W_{\text {free }}$. Consequently, the drug encapsulation efficiency (EE) and drug loading (DL) could be calculated using the following equations (Liu and Gao 2009).

$\mathrm{EE}(\%)=\frac{W_{\text {total }}-W_{\text {free }}}{W_{\text {total }}} \times 100$

$\mathrm{DL}(\%)=\frac{W_{\text {total }}-W_{\text {free }}}{W_{\text {lipid }}} \times 100$,

where $W_{\text {total }}$ is the total amount of drug, $W_{\text {free }}$ is the amount of unencapsulated drug and $W_{\text {lipid, }}$ the weight of the lipid.

\section{FT-IR spectroscopy}

FTIR was performed using a Perkin Elmer system 2000 spectrophotometer to understand if there is any interaction between the drug and excipients. The spectra were obtained at the region $4000-400 \mathrm{~cm}^{-1}$.

\section{Differential scanning calorimetry}

The physical states of the NLCs were characterized using a differential scanning calorimetric (DSC) thermogram analyser (STA 6000 Simultaneous Thermal Analyser, Perkin Elmer, Waltham, MA). $6 \mathrm{mg}$ of each sample (Ifosfamide, excipients and NLCs) was sealed separately in a standard aluminum pan, and purged with pure dry nitrogen gas set at a flow rate of $10 \mathrm{~mL} / \mathrm{min}$, the temperature variation was set at $10{ }^{\circ} \mathrm{C} / \mathrm{min}$ and the heat flow was recorded from 0 to $350{ }^{\circ} \mathrm{C}$.

\section{$X$-ray diffraction $(X R D)$ studies}

The X-ray diffraction (XRD) patterns of NLCs were determined using XRD diffractometry (D8 ADVANCE, Bruker AXS Inc, Madison, WI). The X-ray source was high flux K- $\alpha-1$ radiation from a copper target with a graphite monochromater. The $\mathrm{X}$-ray tube was operated at a potential of $40 \mathrm{kV}$ and a current of $100 \mathrm{~mA}$. The range $(2 \theta)$ of scans was from 0 to $50^{\circ}$ and the scan speed was $2^{\circ}$ per minute at increments of $0.02^{\circ}$. The XRD patterns of Ifosfamide, blank nanoparticles and NLCs were obtained.

\section{In vitro release study}

The in vitro release was carried out using multi-compartment rotating cells with a dialysis membrane (cut off $12,000 \mathrm{Da})$. The donor phase consisted of $2 \mathrm{~mL}$ of formulation in phosphate buffer at $\mathrm{pH}$ 7.4. The receiving phase also consisted of phosphate buffer, $\mathrm{pH}$ 7.4. The receiving phase was completely withdrawn and replaced with fresh medium after fixed time intervals, suitably diluted and analyzed using the HPLC method as described in the Sect. 2.4. The release studies were conducted in the same way in both $\mathrm{pH} 1.2$ and $\mathrm{pH} 6.8$ phosphate buffer solutions.

\section{Stability studies}

NLC formulations were stored in the sealed amber-colored glass vials at $2-8{ }^{\circ} \mathrm{C}$ and $25{ }^{\circ} \mathrm{C}$ under dark environment. The formulations were analyzed for particle size, zeta potential, drug loading and encapsulation efficiency after 1, 3 and 6 months of storage and compared with formulations made initially. In addition, the drug release study of the NLCs stored at $2-8{ }^{\circ} \mathrm{C}$ and $25^{\circ} \mathrm{C}$ for 6 months was performed and compared with the formulations made initially. The experiments were performed in triplicate.

\section{Statistical analysis}

The relationships between responses and formulation variables of all model formulations were treated by Design Expert $^{\circledR}$ 8.0.7.1 software. Statistical analysis, including stepwise linear regression and response surface analysis was conducted. The significant terms $(p<0.05)$ were chosen for final equations. Suitable models consisting of three components include linear, quadratic and special cubic models. The best-fitting mathematical model was selected based upon the comparisons of several statistical parameters, including the coefficient of variation $(\mathrm{CV})$, the multiple correlation coefficients $\left(R^{2}\right)$ and the adjusted multiple correlation coefficient (adjusted $R^{2}$ ).

\section{Results}

\section{Optimization of formulations}

The CCRD-RSM constitutes an alternative approach because it offers the possibility of investigating a high number of variables at different levels with only a limited number of experiments (Ahn et al. 2008). The variables in the Table 1 were chosen taking into account our 
Table 2 Central composite design consisting of experiments for the study of three experimental factors in coded levels with experimental results

\begin{tabular}{|c|c|c|c|c|c|c|}
\hline \multirow[t]{2}{*}{ Formulation } & \multicolumn{3}{|c|}{ Coded value variables } & \multicolumn{3}{|c|}{ Response values } \\
\hline & $\mathrm{X}_{1}$ & $\mathrm{X}_{2}$ & $\mathrm{X}_{3}$ & $\mathrm{EE}(\%)$ & DL $(\%)$ & PS (nm) \\
\hline 1 & -1 & -1 & -1 & 70.2 & 13.2 & 283.3 \\
\hline 2 & +1 & -1 & -1 & 68.3 & 19.2 & 295.2 \\
\hline 3 & -1 & +1 & -1 & 62.1 & 10.3 & 315.2 \\
\hline 4 & +1 & +1 & -1 & 56.2 & 14.5 & 350.4 \\
\hline 5 & -1 & -1 & +1 & 71.3 & 11.2 & 285.6 \\
\hline 6 & +1 & -1 & +1 & 73.4 & 17.1 & 310.4 \\
\hline 7 & -1 & +1 & +1 & 81.2 & 9.2 & 310.2 \\
\hline 8 & +1 & +1 & +1 & 73.1 & 14.3 & 339.3 \\
\hline 9 & -1.682 & 0 & 0 & 69.3 & 7.6 & 335.2 \\
\hline 10 & +1.682 & 0 & 0 & 60.2 & 18.5 & 396.8 \\
\hline 11 & 0 & -1.682 & 0 & 66.4 & 17.7 & 385.6 \\
\hline 12 & 0 & +1.682 & 0 & 69.3 & 11.4 & 380.6 \\
\hline 13 & 0 & 0 & -1.682 & 64.2 & 13.1 & 265.4 \\
\hline 14 & 0 & 0 & 1.682 & 74.6 & 12.8 & 220.2 \\
\hline 15 & 0 & 0 & 0 & 67.8 & 13.9 & 325.4 \\
\hline 16 & 0 & 0 & 0 & 67.2 & 14.6 & 325.8 \\
\hline 17 & 0 & 0 & 0 & 67.9 & 14.8 & 325.2 \\
\hline 18 & 0 & 0 & 0 & 67.1 & 14.2 & 325.4 \\
\hline 19 & 0 & 0 & 0 & 67.3 & 14.4 & 325.4 \\
\hline 20 & 0 & 0 & 0 & 67.4 & 14.2 & 325.8 \\
\hline
\end{tabular}

preliminary experiments. Table 2 showed the experimental results concerning the tested variables on entrapment efficiency (EE), drug loading (DL) and particle size (PS). The three dependent values EE, DL and PS ranged from 56 to $81 \%$ by weight, $7-19 \%$ by weight and $220-396 \mathrm{~nm}$, respectively. A mathematical relationship between factors and parameters was generated by response surface regression analysis using Stat-Ease Design Expert $^{\circledR}$ 8.0.7.1 software. The three-dimensional (3D) response surface graphs for the most statistically significant variables on the evaluated parameters are shown in Fig. 3.

The response surface diagrams showed that the higher the surfactant concentration the higher the EE, and the higher the lipid concentration the higher the DL and larger the PS. In addition, a higher ratio of aqueous phase to organic phase resulted in higher EE, lesser DL and also smaller PS. Furthermore, the PS significantly increased with the increasing aqueous phase ratio indicating that the aqueous phase ratio significantly affected the PS. It was observed that the best fitted model was the quadratic model, and the comparative values of $R, \mathrm{SD}$ and \% $\mathrm{CV}$ along with the regression equation generated for the selected responses are given in Table 3. Only statistically significant $(p<0.05)$ coefficients are included in the equations. A positive value represents an effect that favours the optimization, while a negative value indicating an inverse relationship between the factor and the response (Myers and Montgomery 2002). It is clear from the equation that the factor drug/lipid ratio $\left(X_{1}\right)$ as well as organic/ aqueous phase ratio $\left(X_{2}\right)$ has a negative effect and that concentration of surfactant $\left(X_{3}\right)$ has a positive effect on the response EE. The factor organic/aqueous phase ratio $\left(X_{2}\right)$ and concentration of surfactant $\left(X_{3}\right)$ have a negative effect, and the factor drug/lipid ratio $\left(X_{1}\right)$ has a positive effect on the response DL. As far as PS is concerned, the factor drug/ lipid ratio $\left(X_{1}\right)$ and organic/aqueous phase ratio $\left(X_{2}\right)$ have a positive effect, and the concentration of the surfactant $\left(X_{3}\right)$ has a negative effect. It also shows that the relationship between responses and factors is not always linear. Used at different levels in an analysis or when more than one factor is changed simultaneously, a factor can produce different degrees of response.

Predicted optimum ranges of the independent variables are listed in Table 4. The fitting results indicated that the optimized NLC with high EE, high DL percentage and small PS was obtained with the drug/lipid ratio of 1:3, organic/aqueous phase ratio of $1: 10$ and surfactant concentration of $1 \% \mathrm{w} / \mathrm{v}$, respectively. Table 4 shows that the experimental values of the two batches prepared within the optimum range were very close to the predicted values, with low percentage bias, suggesting that the optimized formulation was reliable and reasonable. It can be concluded that a high desirability value could be obtained with a drug/lipid ratio of $1: 3$, organic/aqueous phase ratio of $1: 10$ and surfactant concentration of $1 \% \mathrm{w} / \mathrm{v}$.

Perturbation plots are presented in Fig. 4 for predicted models to gain a better understanding of the investigated procedure. These types of plots show the effect of an independent factor on a specific response, with all other factors held constant at a reference point (Myers and Montgomery 1995). A steepest slope or curvature indicates sensitiveness of the response to a specific factor. Figure $4 \mathrm{a}$ shows that drug/lipid ratio (Factor A) had the most important effect on EE followed by Factor B and C. Figure $4 \mathrm{~b}$ shows that organic/aqueous phase ratio (Factor B) had the most important effect on DL followed by factor $\mathrm{C}$ and then A. Figure $4 \mathrm{c}$ shows that concentration of surfactant (Factor C) had the most important effect on particle size followed by factor $\mathrm{A}$ and then $\mathrm{B}$.

\section{Characterization}

Particle size, polydispersity index and zeta potential measurement

The mean particle size of NLC was $223 \mathrm{~nm}$ with a polydispersity index of $0.194 \pm 0.025$. A narrow PI means that the colloidal suspensions are homogenous in nature. The 

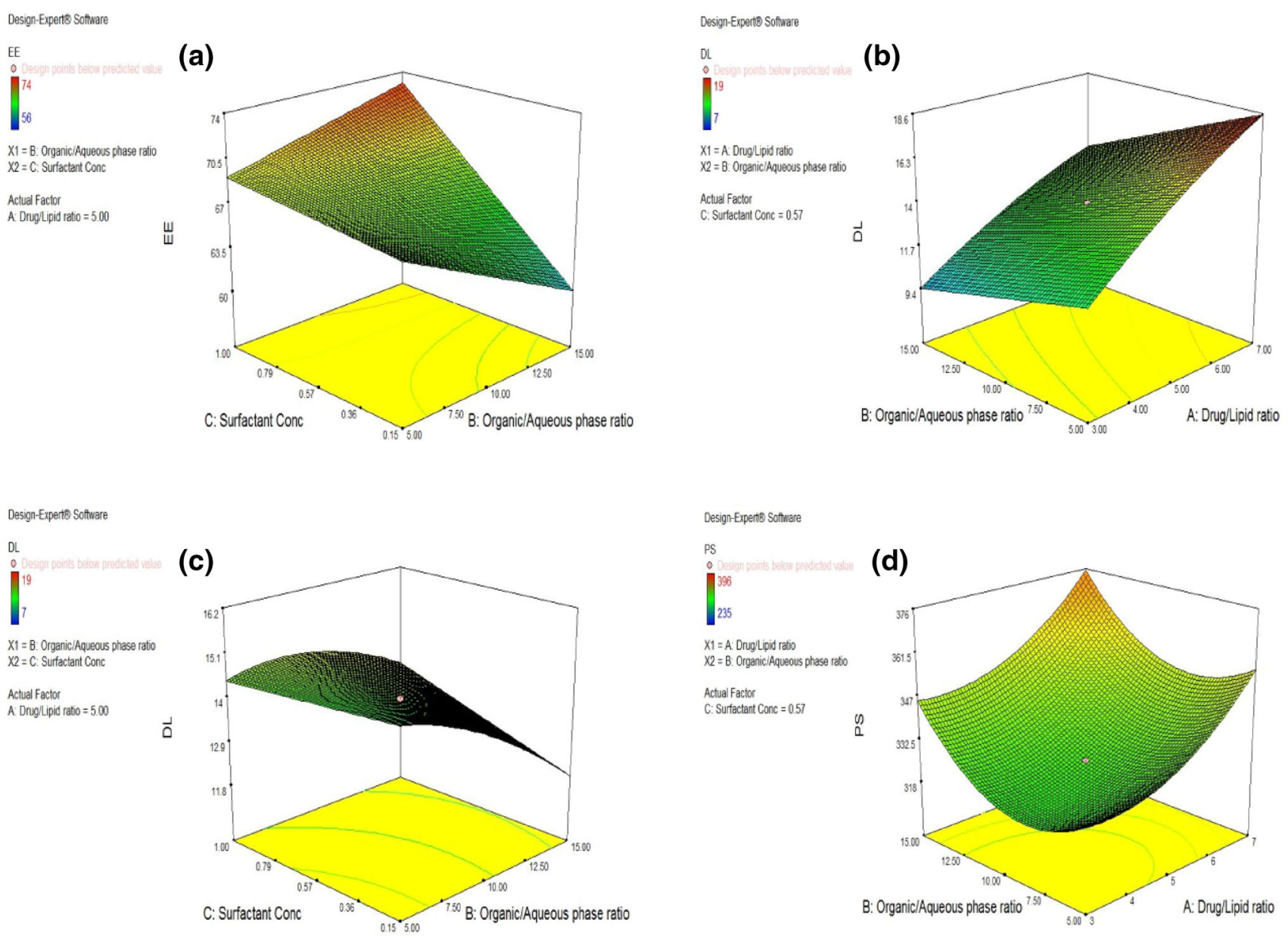

Fig. 3 Three-dimensional (3D) response surface plots showing the effect of the variable on response. a The effect of organic/aqueous phase ratio and surfactant concentration on the entrapment efficiency; b the effect of drug/lipid ratio and organic/aqueous phase ratio on the

drug loading; c the effect of organic/aqueous phase ratio and surfactant concentration on the drug loading; d the effect of drug/ lipid ratio and organic/aqueous phase ratio on the particle size

Table 3 Reduced response models and statistical parameters obtained from ANOVA

\begin{tabular}{|c|c|c|c|c|c|}
\hline Responses & Regression models & Adjusted $R^{2}$ & Model $P$ value & $\% \mathrm{CV}$ & Adequate precision \\
\hline $\begin{array}{r}\text { Entrapment } \\
\text { efficiency }\end{array}$ & $67.45-1.77 X_{1}-0.88 X_{2}+3.65 X_{3}+2.88 X_{2} X_{3}$ & 0.8126 & 0.0001 & 3.28 & 21.28 \\
\hline Drug loading & $\begin{array}{l}14.06+2.89 \mathrm{X}_{1}-1.69 \mathrm{X}_{2}-0.49 \mathrm{X}_{3}-0.37 \mathrm{X}_{1} \mathrm{X}_{2} \\
+0.37 \mathrm{X} 2 \mathrm{X} 3-0.45 \mathrm{X}_{1}^{2}-0.45 \mathrm{X}_{3}^{2}\end{array}$ & 0.8563 & 0.0001 & 2.86 & 10.42 \\
\hline Particle size & $\begin{array}{l}325.72+14.91 X_{1}+9.71 X_{2}-3.62 X_{3}+9.62 X_{1}^{2} \\
\quad+15.63 X_{2}^{2}-31.21 X_{3}^{2}\end{array}$ & 0.8824 & 0.0001 & 3.12 & 14.26 \\
\hline \multicolumn{2}{|c|}{ Acceptance criteria } & $\geq 0.80$ & $<0.05$ & $<4$ & $>10 \%$ \\
\hline
\end{tabular}

zeta potential of the NLC was found to be $-25 \mathrm{mV}$, and it is sufficiently high to form stable colloidal nanosuspension.

\section{Transmission electron microscopy}

In order to provide information on the morphology and size of the optimal Ifosfamide NLC, TEM was used to take photos of the optimal NLC formulation, as shown in Fig. 5. The NLC particles are spherical. The size of the NLC is about $200 \mathrm{~nm}$. The diameter observed by TEM is smaller, while the diameter determined by DLS is above $223 \mathrm{~nm}$. The reason may be that the two methods are based on different sample preparation processes. 
Table 4 Comparison of experimental and predicted values under optimal conditions for final formulation

\begin{tabular}{llllll}
\hline Drug lipid ratio & Organic aqueous phase ratio & Surfactant concentration & Particle size (nm) & $\%$ Entrapment efficiency & $\%$ Drug loading \\
\hline $1: 3$ & $1: 10$ & $1 \%$ & & & 79 \\
Predicted & & & 220 & 7.24 & 6.14 \\
Experimental & & & $3 \%$ & $4 \%$ & $1 \%$ \\
Bias (\%) & & & 223 & 77
\end{tabular}

Acceptance criteria $=6 \%$

Bias was calculated as (predicted value - experimental value)/predicted value $\times 100$

(a)

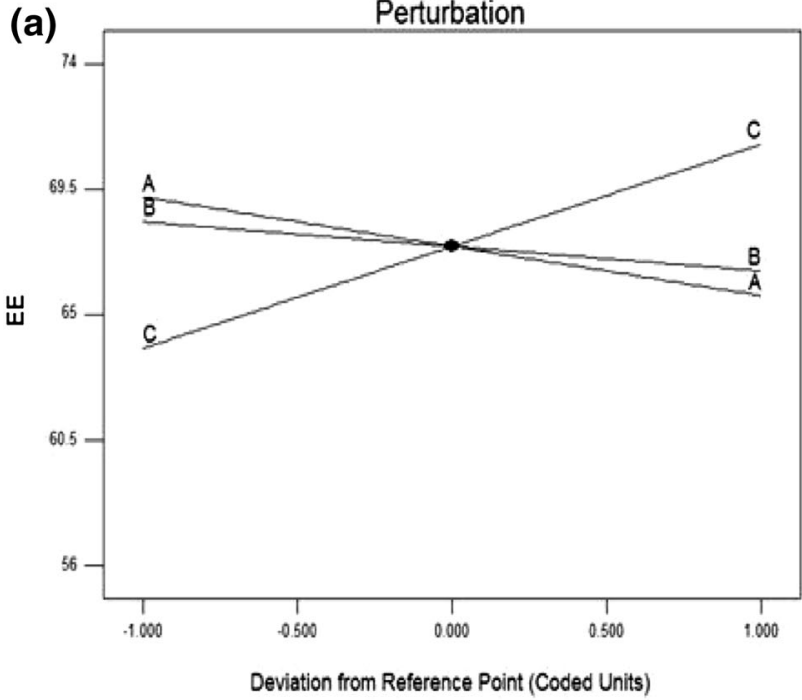

(b)

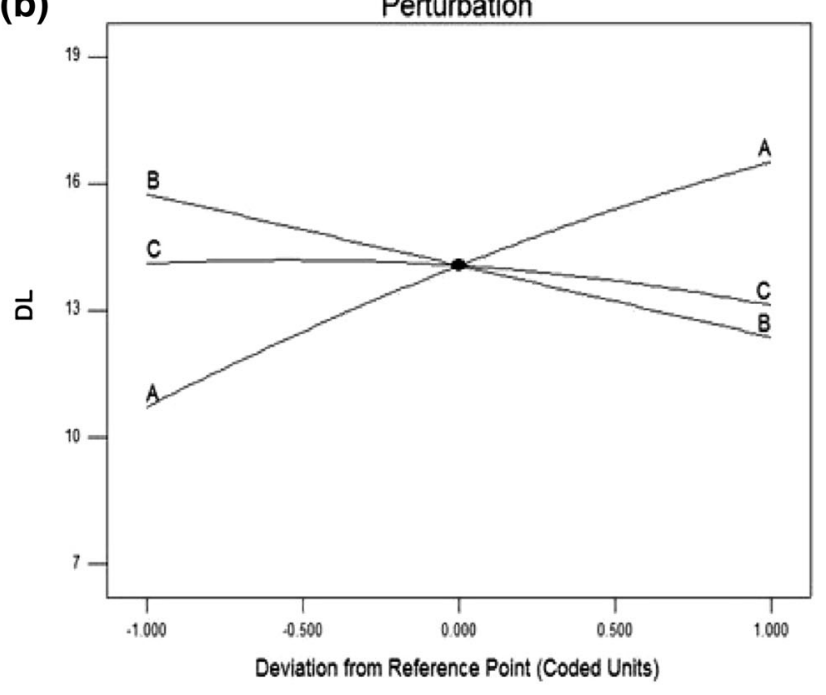

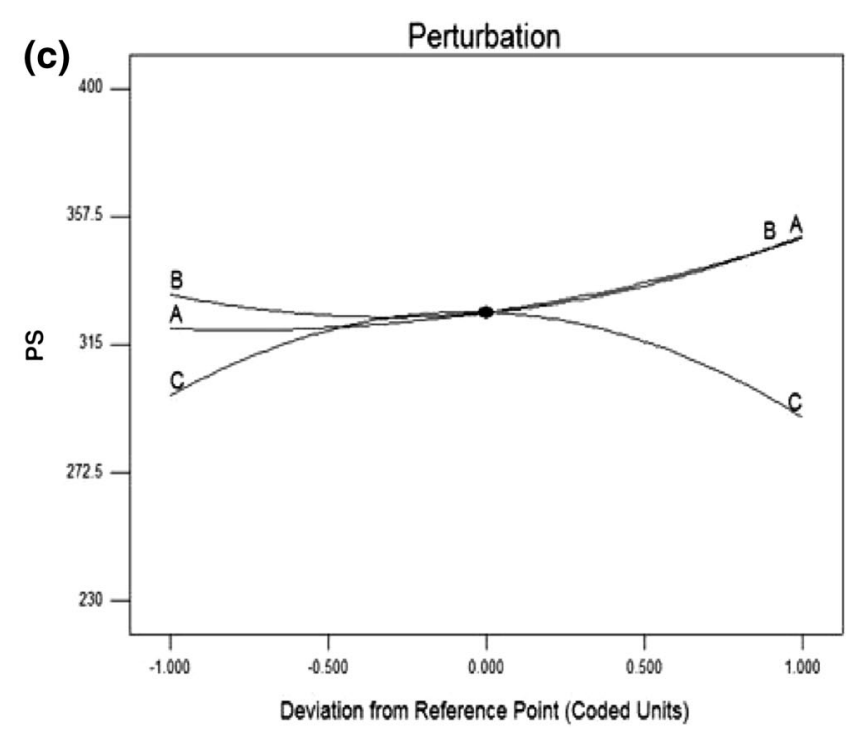

Fig. 4 Perturbation plots showing the effect of each of the independent variables on $\mathbf{a}$ entrapment efficiency, $\mathbf{b}$ drug loading and $\mathbf{c}$ particle size, where $A, B$ and $C$ are drug/lipid ratio, organic/aqueous phase ratio and concentration of surfactant, respectively

\section{Fourier transform infrared spectroscopy (FTIR)}

The FTIR spectra for chitosan, cross-linked chitosan particles with IF, are shown in Fig. 6. Cross-linked drug loaded particles show a peak for $\mathrm{P}=\mathrm{O}$ bond at $1113 \mathrm{~cm}^{-1}$.
Sodium alginate gives a characteristic peak at $1624 \mathrm{~cm}^{-1}$, which shifts to $1651 \mathrm{~cm}^{-1}$ in cross-linked particles, and a new peak appears at $1736 \mathrm{~cm}^{-1}$ after cross-linking with chitosan. A strong band occurs in the region from 3200 to $3500 \mathrm{~cm}^{-1}$, which becomes very strong in the cross- 
Fig. 5 TEM of Ifosfamide nanostructured lipid carrier a magnification $\times 12,000$ and b magnification $\times 46,000$
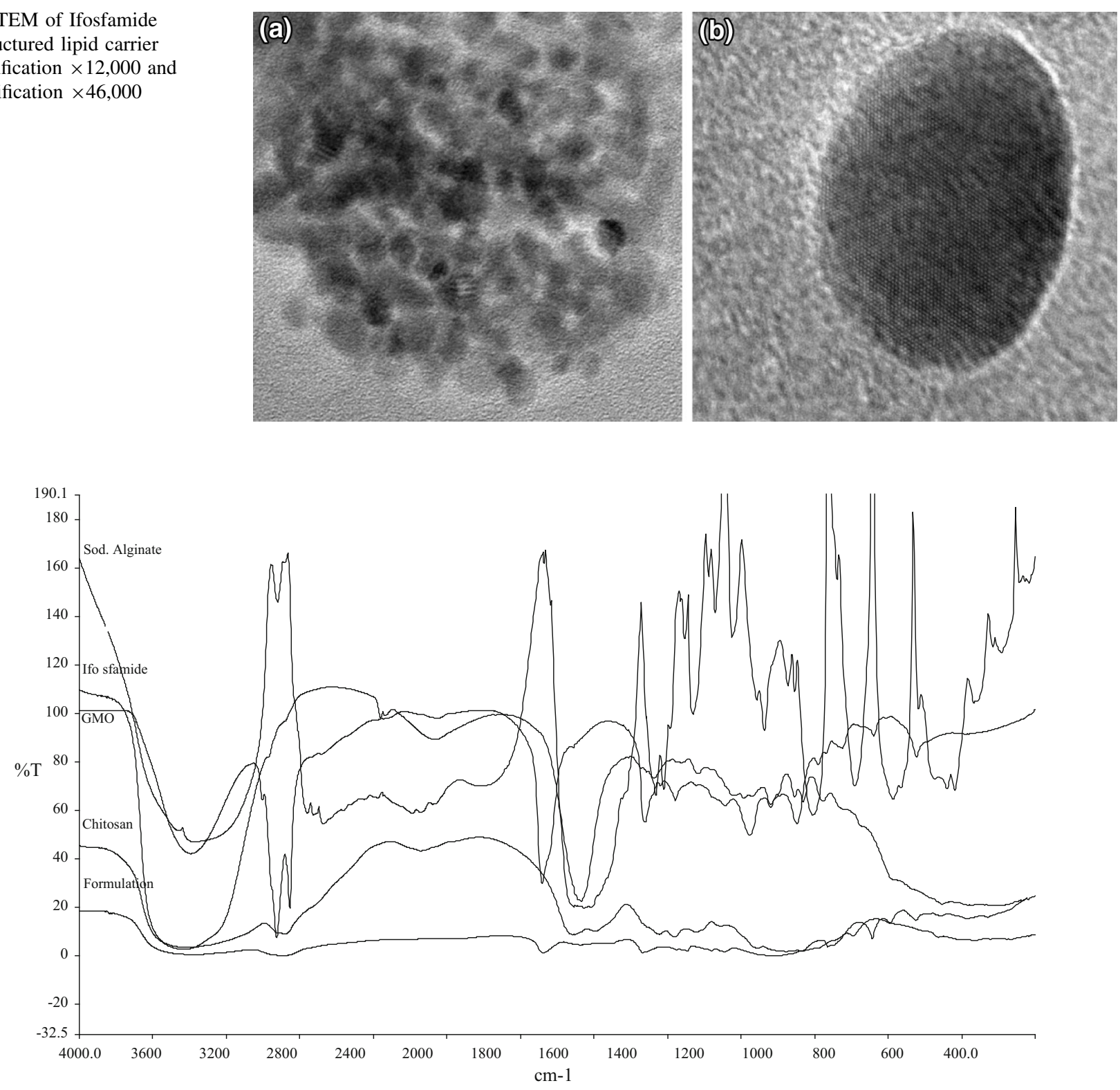

Fig. 6 FTIR of chitosan, sodium alginate, gmo, ifosfamide and ifosfamide nanostructured lipid carrier

linked particles. Peaks at 2924 and $2876 \mathrm{~cm}^{-1}$ were also noticed.

\section{Differential scanning calorimetry}

The DSC analysis was performed for the Ifosfamide, chitosan, sodium alginate, glycerol monooleate and the Ifosfamide formulation to found out the physical state of the drug inside the nanoparticulate formulation. DSC gives a measure of the change in enthalpy. DSC was used to understand the holistic picture of true encapsulation. From Fig. 7, it is observed that the Ifosfamide at $\sim 44{ }^{\circ} \mathrm{C}$ had an endothermic peak of melting point. These characteristic peaks were absent in the formulation. But an endothermic peak at $\sim 110^{\circ} \mathrm{C}$ was observed, due to the melting point of the chitosan crystals. The endothermic peaks of the GMO and sodium alginate were also absent in the final formulation.

\section{$X$-ray diffraction studies}

Figure 8 depicts the comparison of XRD analysis of Ifosfamide, blank NLCs and NLCs. The diffraction pattern exhibited Ifosfamide had two sharp peaks at $2 \theta=14.78^{\circ}$, and $15.16^{\circ}$ and some peaks of lower intensity. These results indicate the crystalline nature of Ifosfamide. The diffraction characterised peaks were absent in the formulation as well as in the blank nanostructured lipid carrier. 
Fig. 7 DSC of ifosfamide, glycerol monooleate, chitosan, nanostructured lipid carrier sodium alginate and ifosfamide

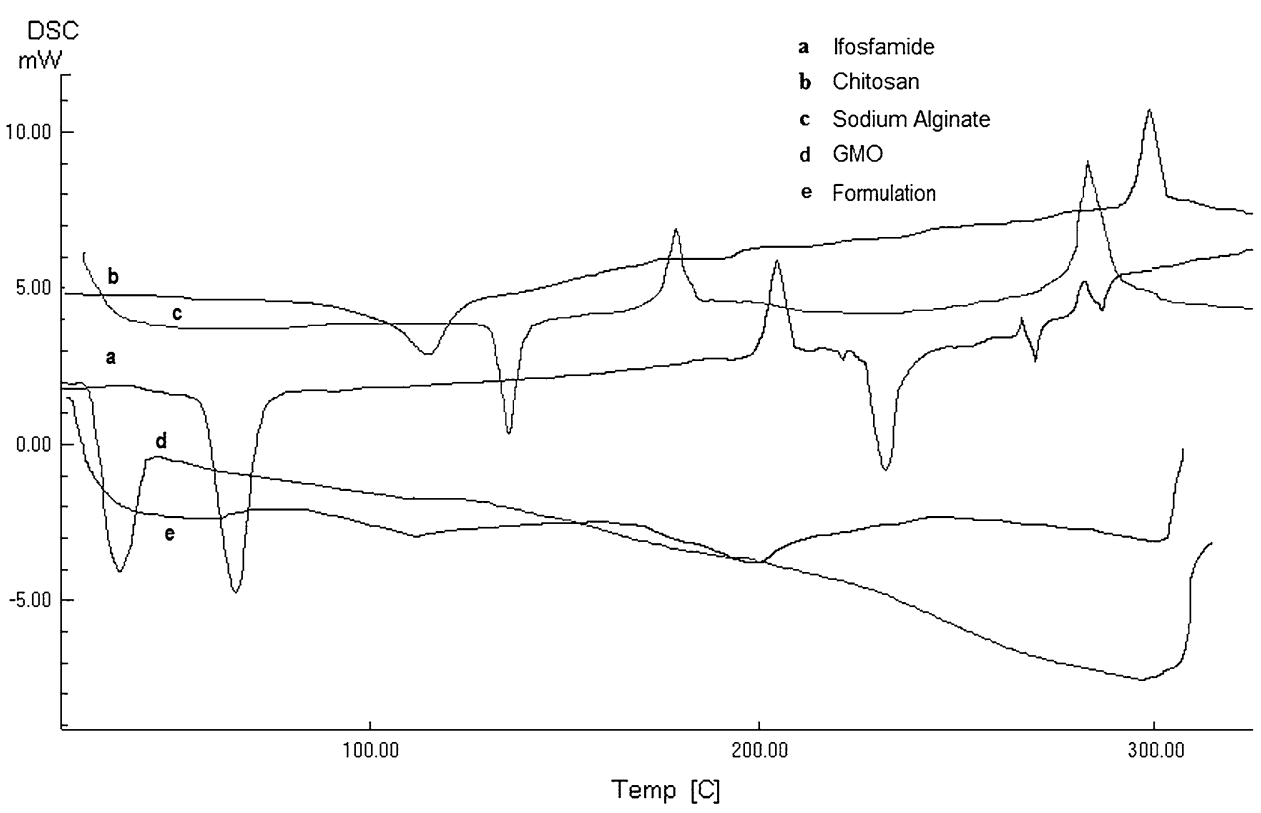

Fig. 8 XRD of a ifosfamide, b blank nanostructured lipid carrier and $\mathbf{c}$ ifosfamide nanostructured lipid carrier

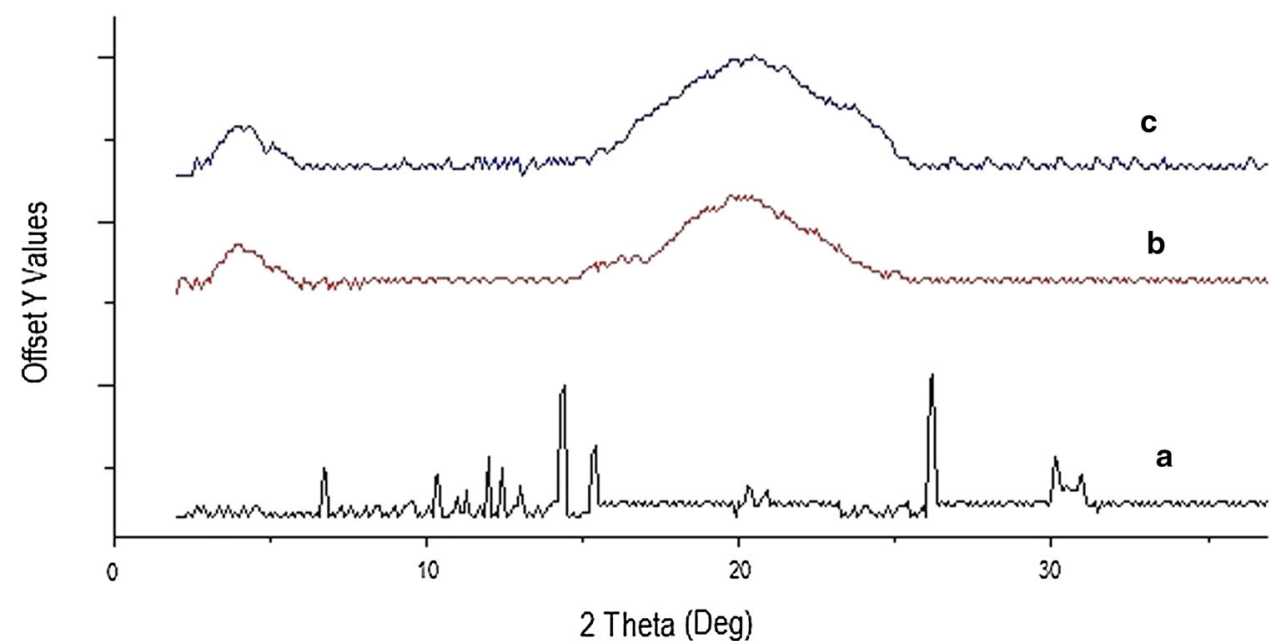

\section{The in vitro release study of optimized Ifosfamide NLC formulation}

The in vitro release curve of the optimal formulation is shown in Fig. 9. The drug release from the formulation was noted to be only 13 and $15 \%$ in $\mathrm{pH} 1.2$ and $\mathrm{pH} 6.8$ phosphate buffer, respectively (Fig. 9a, b).

The delivery system showed an initial burst release with approximately $62 \%$ drug being released at the end of $24 \mathrm{~h}$ in $\mathrm{pH} 7.4$ phosphate buffer solutions. The release of Ifosfamide was gradual thereafter with a plateau being reached after $70 \mathrm{~h}$ (Fig. 9c). Meanwhile, the drug is quickly released from conventional suspension compared with that of NLC (Fig. 9d).

Release data of drug from the nanoformulation was well fitted to first-order reaction, with the $R^{2}$ value of 0.979 and described as follows: $\log Q_{\mathrm{t}}=\log Q_{0}+\mathrm{Kt} / 2.303$

\section{Stability of Ifosfamide loaded nanostructured lipid carrier}

To investigate the effect of storage temperature on the stability of Ifosfamide nanoformulation, the NLC suspensions were stored at $2-8{ }^{\circ} \mathrm{C}$ and $25^{\circ} \mathrm{C}$ in the dark over a period of 180 days. An increase in particle size and decrease in zeta potential, drug loading and entrapment efficiency were observed with storage time at both the storage conditions are shown in Table 5. To evaluate any changes of drug release profile during storage, drug release studies were performed and compared with the initial formulations depicted in Fig. 10. A marginal difference in release rate was observed from both the formulations stored at different storage conditions. A sustained drug release was noticed with both the formulations stored at different storage conditions. 
$\%$ drug release of formulation (pH 1.2)

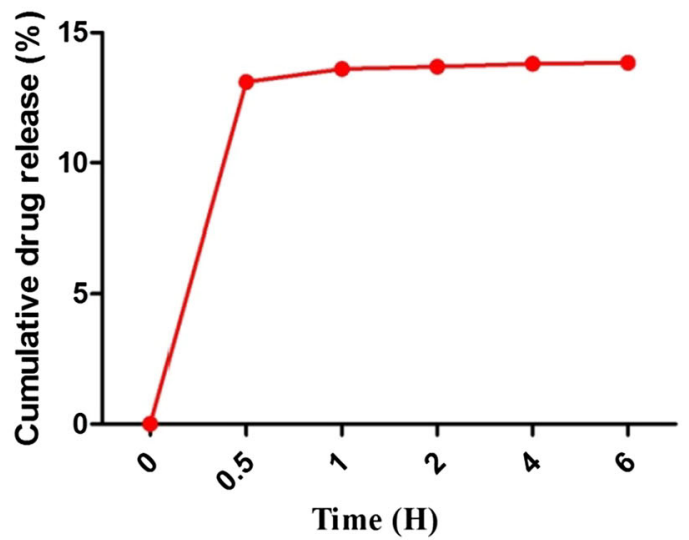

$\%$ drug release of formulation (pH 7.4)

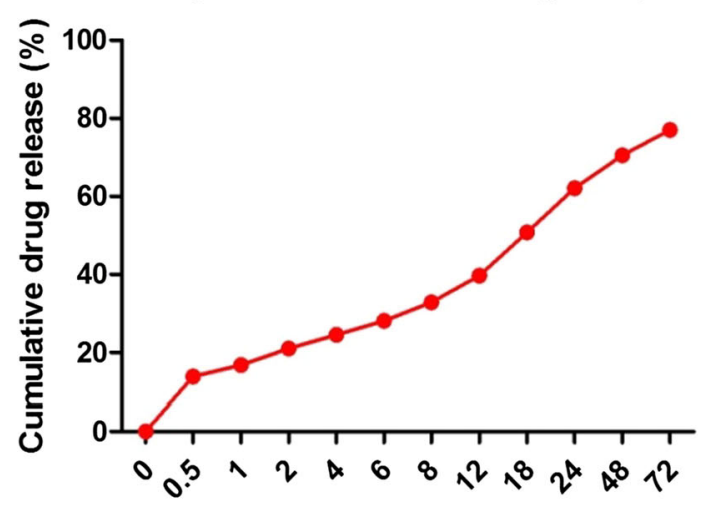

Time (H)

Fig. 9 Cumulative $\%$ drug release of Ifosfamide nanostructured lipid carrier. a \% drug release at the $\mathrm{pH} 1.2, \mathbf{b} \%$ drug release at the $\mathrm{pH} 6.8$, c $\%$ drug release at the $\mathrm{pH} 7.4$ and d Comparison of the \% drug

\section{Discussion}

\section{Formulation of delivery system}

The nanoparticles were prepared by solvent diffusion technique as previously described by Yuan et al. (2007) and $\mathrm{Hu}$ et al. (2008). Glycerol monooleate was used as an internal phase (Trickler et al. 2008). Poloxamer and sodium alginate were used as surfactant and cross linking agents, respectively. Glycerol monooleate being a lipid can incorporate large amount of water in it and form liquid crystalline phase. This property has been used for the developing sustained release formulation of both hydrophobic and hydrophilic drugs (Boyd et al. 2006). The purpose of using oleic acid in the preparation of the nanoparticles was to stabilize the water in oil emulsions (Binks and Lumsdon 2000). Low-molecular weight chitosan was for slow release of Ifosfamide from the NLCs. Chitosan has the higher degree of deacetylation, which makes it more soluble in biological system (Sannan et al.
$\%$ drug release of formulation (pH 6.8)
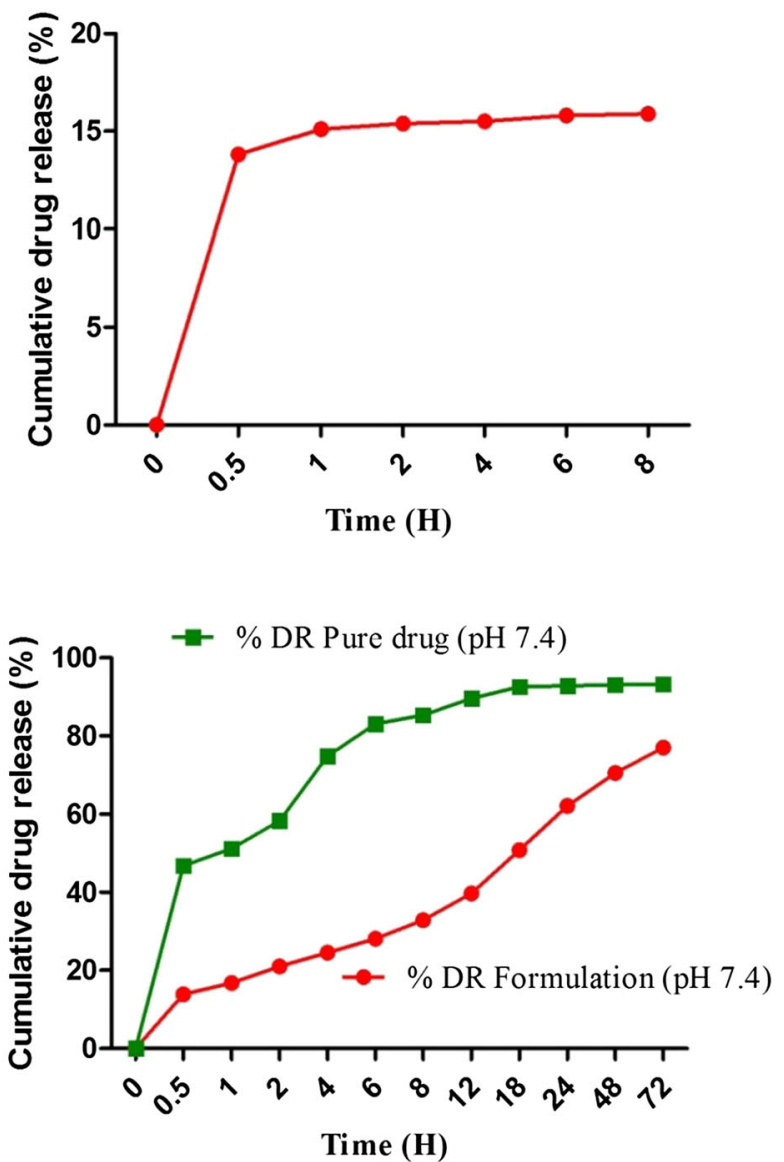

release of Ifosfamide nanostructured lipid carrier and Ifosfamide as a pure drug candidate

1976). The low-molecular-weight chitosan also helps to achieve smaller particle size and better redispersibiliy of particles in water (Trickler et al. 2010). The nanoparticles prepared with chitosan produce positive surface charge. However, the addition of the sodium alginate makes the nanoparticles negatively charged, which could improve the cellular uptake of the nanoparticles. Ifosfamide is a highly hydrophilic drug and, therefore, a high drug loading, using only the lipid phase, is very difficult. Therefore, chitosan is used over nanostructured lipid carriers to enhance the drug loading and to modify the drug-release from these nanoparticles. The Ifosfamide NLC was prepared using three different ratios of lipid viz. 1:3, 1:5 and 1:7. Based on the nanoparticle recovery and $\mathrm{EE}$, the 1:3 ratio was selected as the best ratio than the other two. The other two ratios produced low DL which causes high drug wastage during the preparation process and also produce larger particles. These have been repeatedly tried for three times, for reproducibility and for consistency.

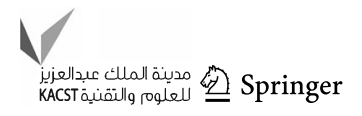




\section{Influence of aqueous phase volume}

The ratio of oil phase and aqueous phase showed a great impact on the EE of NLC. Figure 3a clearly shows that increasing aqueous phase volume results in an increase in EE. This could be due to lesser aggregation of the particles in a larger space. Different studies have shown that the aqueous phase volume has a paramount effect on the formation of nanoparticles. In a recent study, with increased volume of aqueous phase, increase in drug content of particles prepared by homogenization and sonication was observed (Budhian et al. 2007). Aqueous phase volume beyond $10 \mathrm{~mL}$ was not advantageous because of lower concentration of nanodispersion without the increase in EE.

Table 5 Particle size, zeta potential, entrapment efficiency and drug loading capacity of ifosfamide-loaded NLCs stored at $2-8{ }^{\circ} \mathrm{C}$ and $25{ }^{\circ} \mathrm{C}$ over a period of 6 months

\begin{tabular}{lll}
\hline Samples & $2-8{ }^{\circ} \mathrm{C}$ & $25{ }^{\circ} \mathrm{C}$ \\
\hline PS (nm) & & \\
Initial & $223.2 \pm 0.1$ & $223.2 \pm 0.1$ \\
3 months & $228.2 \pm 0.5$ & $230.9 \pm 0.3$ \\
6 months & $230.9 \pm 0.1$ & $237.3 \pm 0.4$ \\
ZP (-mV) & & \\
Initial & $25.7 \pm 2.6$ & $25.7 \pm 2.6$ \\
3 months & $23.6 \pm 3.8$ & $23.5 \pm 2.7$ \\
6 months & $21.9 \pm 2.6$ & $20.5 \pm 2.4$ \\
EE (\%) & & \\
Initial & $81.7 \pm 1.4$ & $78.1 \pm 1.4$ \\
3 months & $80.3 \pm 1.3$ & $74.8 \pm 1.8$ \\
6 months & $78.6 \pm 1.2$ & \\
DL (\%) & & $7.2 \pm 0.2$ \\
Initial & $7.2 \pm 0.2$ & $7.5 \pm 0.2$ \\
3 months & $7.6 \pm 0.1$ & $7.5 \pm 0.1$ \\
6 months & $7.6 \pm 0.1$ & \\
\hline
\end{tabular}

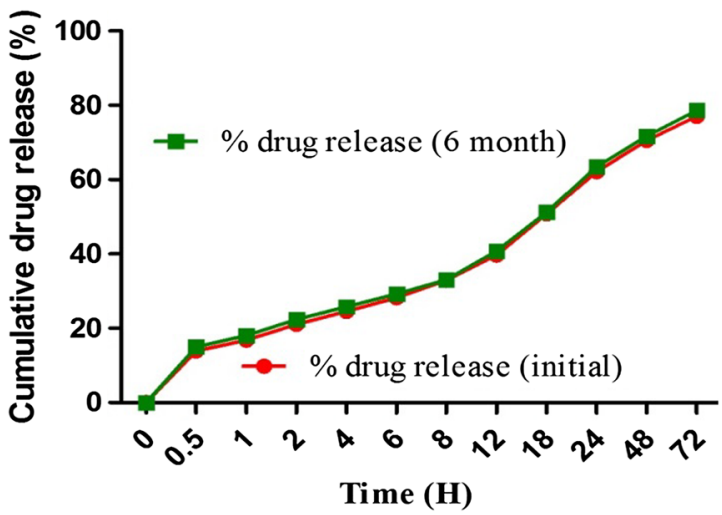

\section{Effect of surfactant concentration}

The type of compound employed for stabilization has a pronounced effect on PS. The mean PS was found to decrease sharply with an increase in concentration of Lutrol $F$ 68 up to $1 \% \mathrm{w} / \mathrm{v}$. The higher surfactant concentration reduces the surface tension and facilitates particle partition. The decrease in the PS is accompanied by a rapid and tremendous increase in the surface area. Thus, the process of primary coverage of the newer surfaces competes with the agglomeration of the uncovered surfaces. Hence, an increase in the surfactant concentration in the primary dispersion results in rapid coverage of the newly formed particle surfaces. There was an optimum concentration, above which an increase in surfactant concentration did not result in a decrease in PS due to a saturation point (Reddy et al. 2006).

The broadness of the size distribution observed at higher surfactant concentrations could be due to the higher viscosity of the continuous phase which disperses the stirring energy (Song et al. 2008). Thus, the PI value increased with increasing surfactant concentrations. Stability (high ZP either positive or negative) increased with increasing concentrations of surfactant. Thus, higher stability was observed with formulations having $1 \% \mathrm{w} / \mathrm{v}$ surfactant. An increase in concentration of surfactant resulted in a slight increase in EE and DL, as shown in Fig. 3a and c.

\section{Influence of drug/lipid matrix ratio}

Increase in matrix content is expected to raise the EE by providing more space to incorporate the drug. Increment of the lipid content also reduces the escaping of drug into the external phase, which accounts for an increase in EE (Shah et al. 2007). EE significantly increased at drug: lipid ratio increasing from $1: 3$ to $1: 7$, whereas with this increasing ratio, an increase in PS was observed. Numerous studies have reported that increasing lipid content results in larger

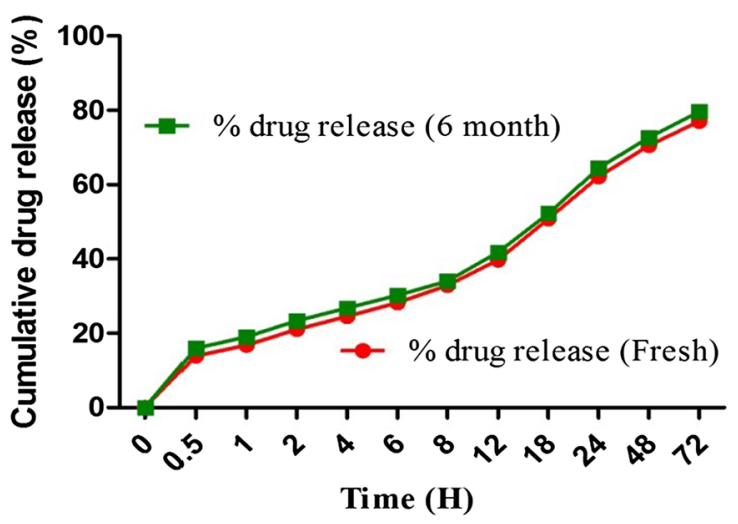

Fig. 10 Comparison of drug release from initial and 6-month-old NLC stored at a $2-8{ }^{\circ} \mathrm{C}$ and $\mathbf{b} 25{ }^{\circ} \mathrm{C}$. Data represent mean $\pm \mathrm{SD}(n=3)$ 
particles and broader PS distribution (Trotta et al. 2003; Mehnert and Mader 2001; Battaglia et al. 2007). Larger PS with an increase in lipid content could be attributed to decrease in emulsifying efficiency and increase in particle agglomeration and increased DL as well, as shown in Fig. $3 b$ and $d$.

\section{Morphological characterization}

\section{Particle size and zeta potential}

Chitosan, when dissolved in acidic medium, bears a net positive charge due to protonation of free amino groups. On addition of sodium alginate, the protonated amino groups of chitosan interact with -OR groups provided by sodium alginate. The hydroxyl ions are ionically bonded to the amino groups, which causes deprotonation of amino groups (Bhumkar and Pokharkar 2006). This leaves negatively charged phosphoric ions, which have not been utilized for neutralization of positive charge on chitosan, to provide a net negative charge on to the particles. Adsorption of the negatively charged phosphate ions on to chitosan particles could be the underlying mechanism of these particles bearing a negative charge. It has been previously shown that negatively charged particles can be taken up by the Caco-2 cells (Gaumet et al. 2009). Therefore, the purpose of adding sodium alginate to glycerol mono oleate chitosan nanoparticles was to achieve crosslinking as well as changing surface charge so that it can be taken up by the cells in the GI tract. The drug loaded particles had a higher zeta potential. This could be attributed to the free Ifosfamide adsorbed at the surface of the particles. The secondary nitrogen attached to the phosphorus atom of the phosphamide group in Ifosfamide could be protonated to give it a net positive charge, which causes an increase in the zeta potential of drug loaded particles.

Figure 5 shows that the Ifosfamide NLC has smooth spherical shaped appearance. The surface of formulated nanoparticles depends on two factors: (a) a saturated solution of polymer chitosan produced smooth and high yield nanoparticles. The undissolved polymer produced irregular and rod-shaped particles; (b) the diffusion rate of solvent is too fast and the solvent may diffuse into the aqueous phase before stable nanoparticles are developed or formed causing the aggregation of nanoparticle preparation. In this preparation the polymer was completely saturated and the diffusion rate of solvent was minimal leading to the formation of smooth, spherical and individually homogeneously distributed particles and has no evidence of collapsed particles. Smooth surface reveals complete removal of solvent from the formulated nanoparticles.

\section{FTIR spectroscopy}

The sharp peak occurring at $1624 \mathrm{~cm}^{-1}$ indicates the presence of sodium alginate, which shifts slightly to $1651 \mathrm{~cm}^{-1}$ and a peak appears at $1736 \mathrm{~cm}^{-1}$ indicating the bending vibration of amino groups present in chitosan after cross-linking to sodium alginate, A characteristic band between 3500 and $3200 \mathrm{~cm}^{-1}$, which is due to $\mathrm{O}-\mathrm{H}$ and N$\mathrm{H}$ stretching, was seen in chitosan. This band is further intensified in the cross-linked particles due to interaction between hydroxyl ions of sodium alginate and amino groups of chitosan. This further confirms the cross-linking of sodium alginate with chitosan in the formulation. The cross linking between the sodium alginate and chitosan molecule happens either by deprotonation or ionic cross linking (Bhumkar and Pokharkar 2006). In the process of deprotonation, there is a formation of hydrogen bonds between the positively charged amino groups of chitosan and negatively charged hydroxyl groups donated by sodium alginate. Thus, this extensive hydrogen bonding intensifies the peak at $3500-3200 \mathrm{~cm}^{-1}$. A new peak appears in crosslinked particles at $1113 \mathrm{~cm}^{-1}$, which could be attributed to $\mathrm{P}=\mathrm{O}$ bond that is derived from sodium alginate. Peaks at 2924 and $2876 \mathrm{~cm}^{-1}$ were observed, which denote $\mathrm{C}-\mathrm{H}$ stretching in alkanes. GMO is an ester of glycerol and oleic acid. Oleic acid is a straight chain carboxylic acid composed of 18 carbons. Furthermore, oleic acid present in the formulation also had alkyl chains. $\mathrm{C}-\mathrm{H}$ stretching in alkanes is seen in the range of $3000-2850 \mathrm{~cm}^{-1}$. Thus, the peaks at 2924 and $2876 \mathrm{~cm}^{-1}$ could be attributed to oleic acid and GMO present in the formulation.

Comparing the FTIR spectra of pure polymer, lipid, drug and formulation, we confirmed that there is no significant interaction between drug and polymer and has good chemical stability.

\section{Differential scanning calorimetry}

The endothermic peak of GMO at $30{ }^{\circ} \mathrm{C}$ shifted to $26{ }^{\circ} \mathrm{C}$ in Ifosfamide-loaded NLCs. This shift might be due to formation of nanoparticles with presence of drug (Ifosfamide loaded NLCs). Reduced peak areas probably indicate a reduction of lipid crystallinity in the NLCs, which should be due to the lipid mixture (less solid lipid crystals). In case of NLC, the nanoparticle matrix was composed of mixture of lipids (solid and liquid lipids) instead of only solid lipid. Although the mixture of solid and liquid lipids were solid at room temperature, the chemical characteristics of the mixture differs with that of the solid lipid when taken alone. Either the liquid lipid was molecularly dispersed in the solid lipid or the nanodroplets of the liquid lipid were dispersed in the solid lipid. The absence of the detectable crystalline endothermic peak of the Ifosfamide, GMO and 
sodium alginate in the formulation clearly indicated that Ifosfamide, encapsulated in the nanoparticulate system, was in the form of amorphous or in solid-state solubilized form in the polymeric matrix (Fig. 7).

\section{$X$-ray diffraction studies}

In order to investigate the changes of the nanoparticle crystalline structure, X-ray diffraction experiments were performed. The X-ray diffraction patterns of ifosfamide, blank NLCs and NLCs are displayed in Fig. 8. The diffractogram of ifosfamide had two sharp peaks at $2 \theta=14.78^{\circ}, 15.16^{\circ}$ and some peaks of lower intensity. On the contrary, these sharp peaks disappeared from that of the NLC formulation, indicating the amorphism of ifosfamide in nanoparticles. Compared to physical mixture the peak intensities of formulaton is weaker, suggesting that the degree of the crystallinity is lower in the nanoparticle than in the raw material. These results indicate that incorporation of ifosfamide to the lipid matrix leads to less ordered crystals in NLC and confirms the deduction in DSC analysis.

\section{In vitro drug release}

The in vitro release studies of Ifosfamide nanoparticles were carried out at $\mathrm{pH} 1.2,6.8$ and 7.4 to correspond to the pattern of release of Ifosfamide at the intestinal $\mathrm{pH}$ (Fig. 9). The initial burst release in the delivery system could be accounted for by any free or surface-bound drug. To investigate the mechanism of drug release from the delivery system, the cumulative drug release was plotted against time. The linear nature of this plot indicates the first-order release with the rate of drug release depending on its concentration. At $\mathrm{pH} 1.2$ and 6.8, Ifosfamide showed a different release pattern compared with that shown at $\mathrm{pH}$ 7.4. There was a reduction in the amount of drug released. The quantity of drug released from the nanoparticle at the $\mathrm{pH} 1.2$ was found to be very low. In the first $6 \mathrm{~h}$, only $13 \%$ of drug has been released, as represented in Fig. 9a. Drug release experiment at the $\mathrm{pH}$ 6.8 shows $15 \%$ drug release at the end of the sixth hour, as represented in Fig. 9b. The delivery system showed an initial burst release with approximately $62 \%$ drug being released at the end of $24 \mathrm{~h}$. The release of Ifosfamide was gradual thereafter with a plateau being reached after $72 \mathrm{~h}$ (Fig. 9c). It is obvious that the decreased percentage of drug release was due to the formation of a more compact wall around the drug by the polymer and the strong crosslinking of chitosan and sodium alginate that would lead to reduction in diffusion of drug from the delivery system. Percentage drug release of a pure drug candidate was performed, and the release was $50 \%$ at first hour and almost $75 \%$ was noted at the fourth hour, as represented in Fig. 9d.

This reduction in the release of drug from the delivery systems could be explained on the basis of interaction between chitosan and sodium alginate. The stronger crosslinking could lead to reduction in diffusion of drug from the matrix and hence decrease in drug release.

\section{Conclusion}

A solvent diffusion method in an aqueous system was employed to prepare the NLC. The Ifosfamide NLC was optimized using the CCRD-RSM by fitting a quadratic model to the response data. The experimental values of the NLC prepared under the optimum conditions were mostly close to the predicted values. Ifosfamide NLC under the optimized conditions gave rise to the EE of $77 \%$, DL of $6.14 \%$, PS of $\sim 223 \mathrm{~nm}$ and $\mathrm{ZP}$ value of $-25 \mathrm{mV}$. TEM showed that the NLC particles are spherical, with drug loaded uniformly on the surface of and inside the NLC. The drug release behavior from the NLC exhibited a biphasic pattern with a burst release at the initial stage and sustained release subsequently. The drug release experiments in the NLC in vitro exhibited a sustained release over $72 \mathrm{~h}$. These results indicated that the NLC obtained in this study could potentially be exploited as a carrier with an initial dose and prolonged plasma level in vivo. The proposed Ifosfamide NLC illustrates an effective way to prolong a drug release. The developed nanoparticles are safer and are the need of the hour for pharmaceutical industry as an alternative drug delivery system in chemotherapy.

Acknowledgments Authors are thankful to BASF (Mumbai, India) for the gist sample of Lutrol F 68 and various excipients as well.

Conflict of interest The authors declare that they have no competing interests.

Open Access This article is distributed under the terms of the Creative Commons Attribution License which permits any use, distribution, and reproduction in any medium, provided the original author(s) and the source are credited.

\section{References}

Ahn JH, Kim YP, Lee YM, Seo EM, Lee KW, Kim HS (2008) Optimization of microencapsulation of seed oil by response surface methodology. Food Chem 107:98-105

Alexander RL, Behme RJ, Brooke D, Scott JA (1993) Lyophilized ifosfamide compositions, US5227373 A, Jul 13

Battaglia L, Trotta M, Gallarate M, Carlotti ME, Zara GP, Bargoni A (2007) Solid lipid nanoparticles formed by solvent-in-water emulsion-diffusion technique: development and influence on insulin stability. J Microencapsul 7:660-672 
Bhumkar DR, Pokharkar VB (2006) Studies on effect of pH on crosslinking of chitosan with sodium tripolyphosphate: a technical note. AAPS Pharm Sci Tech 7:45-50

Binks BP, Lumsdon SO (2000) Catastrophic phase inversion of waterin-oil emulsions stabilized by hydrophobic silica. Langmuir $16: 2539-2547$

Box GEP, Hunter JS (1957) Multi-factor experimental design for exploring response surfaces. Ann Math Stat 28:195-241

Boyd BJ, Whittaker DV, Khoo S (2006) Lyotropic liquid crystalline phases formed from glycerate surfactants as sustained release drug delivery systems. Int J Pharm 309:218-226

Budhian A, Siegel SJ, Winey KI (2007) Haloperidol-loaded PLGA nanoparticles: systematic study of particle size and drug content. Int J Pharm 2:367-375

Chen CC, Tsai TH, Huang ZR, Fang JY (2010) Effects of lipophilic emulsifiers on the oral administration of lovastatin from nanostructured lipid carriers: physicochemical characterization and pharmacokinetics. Eur J Pharm Biopharm 3:474-482

Das S, Chaudhury A (2011) Recent advances in lipid nanoparticle formulations with solid matrix for oral drug delivery. AAPS Pharm Sci Tech 12:62-76

Gaumet M, Gurny R, Delie F (2009) Localization and quantification of biodegradable particles in an intestinal cell models: the influence of particle size. Eur J Pharm Sci 36:465-473

Hu FQ, Zhang Y, Du YZ, Yuan H (2008) Nimodipine loaded lipid nanospheres prepared by solvent diffusion method in a drug saturated aqueous system. Int J Pharm 2:146-152

Kassama LS, Shi J, Mittal GS (2008) Optimization of supercritical fluid extraction of lycopene from tomato skin with central composite rotatable design model. Sep Purif Technol 60:278-284

Liu H, Gao C (2009) Preparation and properties of ionically crosslinked chitosan nanoparticles. Polym Adv Technol 20:613-619

Mehnert W, Mader K (2001) Solid lipid nanoparticles: production, characterization and applications. Adv Drug Deliv Rev 47:165-196

Muller RH, Radtke M, Wissing SA (2002a) Nanostructured lipid matrices for improved microencapsulation of drugs. Int J Pharm 2:121-128

Muller RH, Radtke M, Wissing SA (2002b) Solid lipid nanoparticles (SLN) and nanostructured lipid carriers (NLC) in cosmetic and dermatological preparations. Adv Drug Deliv Rev 54:131-155

Myers RH, Montgomery DC (1995) Response surface methodology. Wiley, New York

Myers RH, Montgomery DC (2002) Response surface methodology: process and product optimization using designed experiments. Wiley, New York
Pandit AA, Dash AK (2011) Surface modified lipid nanoparticulate formulation for ifosfamide: development and characterization. Nanomedicine 8:1397-1412

Radtke M, Souto EB, Muller RH (2005a) Nanostructured lipid carriers: a novel generation of solid lipid drug carriers. Pharm Technol Eur 17:45-50

Radtke M, Souto EB, Muller RH (2005b) Nanostructured lipid carriers: a novel generation of solid lipid drug carriers. Pharm Technol Eur 17:45-50

Reddy LH, Vivek K, Bakshi N, Murthy RS (2006) Tamoxifen citrate loaded solid lipid nanoparticles (SLN): preparation, characterization, in vitro drug release, and pharmacokinetic evaluation. Pharm Dev Technol 2:167-177

Sannan T, Kurita K, Lwakura Y (1976) Effect of deacetylation on solubility. Die Makromolekulare Chemie 12:3589-3600

Selvamuthukumar S, Velmurugan R (2012) Nanostructured lipid carriers: a potential drug carrier for cancer chemotherapy. Lipids Health Dis 11:159

Shah KA, Date AA, Joshi MD, Patravale VB (2007) Solid lipid nanoparticles (SLN) of tretinoin: potential in topical delivery. Int J Pharm 2:163-171

Song X, Zhao Y, Hou S, Xu F, Zhao R, He J, Cai Z, Li Y, Chen Q (2008) Dual agents loaded PLGA nanoparticles: systematic study of particle size and drug entrapment efficiency. Eur J Pharm Biopharm 2:445-453

Trickler WJ, Nagvekar AA, Dash AK (2008) A novel nanoparticle formulation for sustained paclitaxel delivery. AAPS Pharm Sci Tech 9:486-493

Trickler WJ, Khurana J, Nagvekar AA (2010) Chitosan and glycerol monooleate nanostructure containing germcitabine: potential delivery system for pancreatic cancer treatment. AAPS Pharm Sci Tech 1:392-401

Trotta M, Debernardi F, Caputo O (2003) Preparation of solid lipid nanoparticles by a solvent emulsification-diffusion technique. Int J Pharm 2:153-160

Westesen K, Bunjes H, Koch MHJ (1997) Physicochemical characterization of lipid nanoparticles and evaluation of their drug loading capacity and sustained release potential. J Control Release 48:223-236

Yuan H, Wang LL, Du YZ, You Hu FQ, Zeng S (2007) Preparation and characteristics of nanostructured lipid carriers for controlreleasing progesterone by melt-emulsification. Colloids Surf B Biointerfaces 2:174-179 